\title{
OPTICAL MEASUREMENTS OF RAT MUSCLE SAMPLES UNDER TREATMENT WITH ETHYLENE GLYCOL AND GLUCOSE
}

\author{
LUÍS OLIVEIRA $^{*, \dagger,+\dagger}$, MARIA INÊS CARVALHO ${ }^{\S}$, \\ ELISABETE NOGUEIRA ${ }^{*} \sharp$ and VALERY V. TUCHIN ${ }^{\boldsymbol{\top}, \|, * *}$ \\ *Physics Department-Polytechnic of Porto \\ School of Engineering, Rua Dr. António Bernardino \\ de Almeida, 431, 4200-072 Porto, Portugal \\ ${ }^{\dagger}$ University of Porto Rua Dr. Roberto Frias \\ 4200-465 Porto, Portugal \\ $\$$ CIETI-Centre of Innovation in Engineering \\ and Industrial Technology, ISEP, Rua Dr. António Bernardino \\ de Almeida, 431, 4200-072 Porto, Portugal

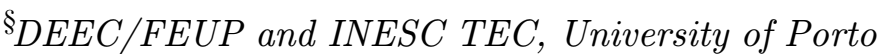 \\ Rua Dr. Roberto Frias, 4200-465 Porto, Portugal \\ "Research-Educational Institute of Optics \\ and Biophotonics, Saratov State University \\ 83 Astrakhanskaya Str., Saratov 410012, Russia \\ "Laboratory of Laser Diagnostics of Technical \\ and Living Systems, Institute of Precise Mechanics \\ and Control RAS, Saratov 410028, Russia \\ **Optoelectronics and Measurement Techniques Laboratory \\ P. O. Box 4500, University of Oulu, FIN-90014, Oulu, Finland \\ †lmo@isep.ipp.pt
}

Received 31 October 2012

Accepted 17 January 2013

Published 20 March 2013

\begin{abstract}
With the objective to study the variation of optical properties of rat muscle during optical clearing, we have performed a set of optical measurements from that kind of tissue. The measurements performed were total transmittance, collimated transmittance, specular reflectance and total reflectance. This set of measurements is sufficient to determine diffuse reflectance and absorbance of the sample, also necessary to estimate the optical properties. All the performed measurements and calculated quantities will be used later in inverse Monte Carlo (IMC) simulations to determine the evolution of the optical properties of muscle during treatments with
\end{abstract}

This is an Open Access article published by World Scientific Publishing Company. It is distributed under the terms of the Creative Commons Attribution 3.0 (CC-BY) License. Further distribution of this work is permitted, provided the original work is properly cited. 
ethylene glycol and glucose. The results obtained with the measurements already provide some information about the optical clearing treatments applied to the muscle and translate the mechanisms of turning the tissue more transparent and sequence of regimes of optical clearing.

Keywords: Tissue optical clearing; optical transmittance; optical reflectance; optical absorbance; agent diffusivity.

\section{Introduction}

The importance of performing optical measurements from biological tissues is very high to evaluate how the tissues respond to light propagation with the objective of creating and improving optical based clinical procedures. ${ }^{1,2}$ Several classes of optical measurements can be obtained from ex vivo tissue samples: transmittance, absorbance, reflection, etc. ${ }^{3}$ If those measurements are to be made while tissues undergo optical clearing treatments, the optical measurements can be performed in a manner to evaluate the time-dependence of the optical response of that tissue samples. The optical clearing effect can also be evaluated with imaging techniques as some researchers have already observed and studied. ${ }^{4-7}$ The optical clearing technique is performed by immersing the biological tissues in chemical agents that can be sugars or alcohols in most cases. When immersing the tissue in such agents an osmotic pressure is created around the tissue that originates tissue dehydration which also precedes agent diffusion into the tissue. Tissue dehydration and refractive index matching inside the tissue due to agent inclusion are the mechanisms involved in optical clearing. ${ }^{8-10}$ Ultimately, these two mechanisms will reduce light scattering inside the tissue. The reduction of light scattering in the tissue may produce changes in the optical properties of the tissue, namely the scattering coefficient, the absorption coefficient and anisotropy factor. ${ }^{11}$ If we desire to characterize the optical clearing effect created by a particular agent and to make a precise distinction between effects created by different agents, we should study the variation produced in the optical properties of biological tissues by those agents.

With the objective of studying the timedependence of the optical response of muscle to treatment with ethylene glycol (EG) and glucose we have performed a set of measurements.

The skeletal muscle can be described as a set of muscle fiber bundles distributed through the interstitial fluid. The muscle contains approximately $75 \%$ of water ${ }^{12}$ that is mostly concentrated in the interstitial space, thus interstitial fluid has a low refractive index $(\sim 1.35)$. On the other hand the dried tissue, which is composed by filaments of actin and myosin (from muscle fibers), corresponds to approximately $25 \%$ of the muscle and has a higher refractive index (1.584). ${ }^{13,14}$ Such distribution creates a step refractive index profile inside the muscle, even muscle fibers normally are hydrated and thus have a lower index of refraction, $\sim 1.46-1.5 .^{14,16}$ Such magnitude in the step of the refractive index profile of the muscle can be greatly reduced with an optical clearing treatment as we have already studied at collimated transmittance measurements. ${ }^{13}$

In the present work we will present and discuss the measurements of total transmittance $\left(T_{t}\right)$, collimated transmittance $\left(T_{c}\right)$, specular reflectance $\left(R_{s}\right)$ and total reflectance $\left(R_{t}\right)$, measured from muscle samples under treatment with the above mentioned optical clearing agents (OCAs). Additionally we will describe also the calculations of absorbance and diffuse reflectance that are necessary for the estimations of the optical properties of the tissues. These measurements and calculations are to be used later to determine the variation of the optical properties of the muscle during the optical clearing treatments applied.

\section{Experimental Method}

Due to the use (or not) of an integrating sphere in the different measuring assemblies, we have selected two designations for the measurements:

A. Integrated measurements - that include total transmittance and total reflectance;

B. Non-integrated - that include collimated transmittance and specular reflectance.

In all cases we have used a Tungsten Halogen lamp with a broad spectrum and a spectrometer to 
measure the spectra. The lamp is the HL-2000 model and the spectrometer is the AvaSpec-2048USB2 model with UA grating set for $200-1100 \mathrm{~nm}$ and $50 \mu \mathrm{m}$ slit, both from Avantes Corporation ${ }^{\mathrm{TM}}$.

\subsection{Tissue samples}

The tissue samples used in this experimental study were obtained from the abdominal wall muscle of rat (Species Wistar Han). All the eight muscle samples used in these studies were obtained from a single animal to guarantee the maximum similarity in their physiology.

After the animal sacrifice, the entire abdominal wall muscle was dissected from the animal and a muscle block was available to prepare the eight samples to use in the experimental studies. All the samples used in measurements were prepared with an approximate circular form with approximately $10 \mathrm{~mm}$ in diameter and $0.5 \mathrm{~mm}$ thickness. The thickness was guaranteed for all samples by the cryostat used to slice them from the muscle block.

\subsection{Optical clearing agents}

The OCAs that were used in this study were EG and glucose. The solution of EG was prepared with $99 \%$ of this agent and $1 \%$ of water and for the glucose solution we have dissolved glucose in water to produce a solution containing $40 \%$ of glucose.

We have measured the refractive index of these solutions, obtaining 1.4280 for EG and 1.3850 for glucose $40 \% .^{9}$ These solutions are recognized as useful OCAs as we have already observed in our previous studies ${ }^{9,15}$ and also as verified by other researchers. ${ }^{16-19}$

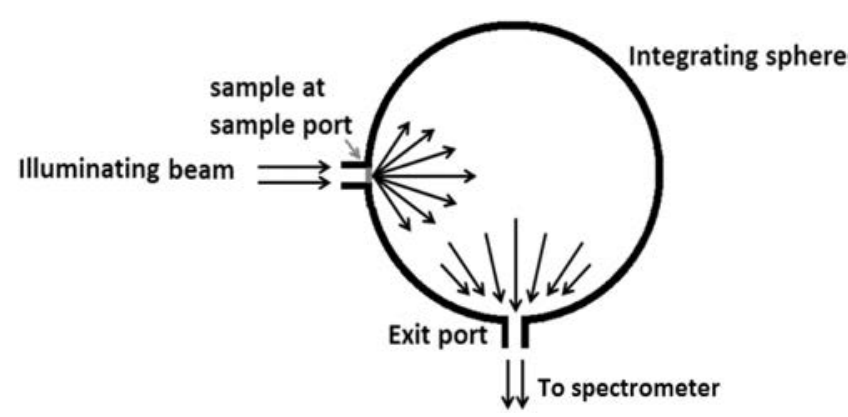

(a)

\subsection{Optical measurements}

In the present study, instead of measuring only the collimated transmittance as before, ${ }^{9}$ we have selected 4 types of measurements for later use in the determination of the optical properties of the muscle and their variation while in optical clearing. The measurements performed were total transmittance, total reflectance, collimated transmittance and specular reflectance. One muscle sample was used in each individual measurement. Additionally, we have also calculated absorbance and diffuse reflectance for each of the optical clearing treatments.

\subsubsection{Integrated measurements - total transmittance and total reflectance}

We will now describe the measuring assemblies necessary to obtain the above designated integrated measurements. In both assemblies presented in this sub-section, one integrating sphere was used to produce total transmitted and total reflected spectra from the muscle samples. The measured spectra were used to calculate total transmittance and total reflectance for the muscle samples with Eqs. (1) and (2) below.

To obtain the total transmittance spectra from the samples, we used the measuring assembly represented in Fig. 1(a) and to obtain the total reflectance spectra we used the measuring assembly represented in Fig. 1(b).

Considering the total transmittance assembly represented in Fig. 1(a), we first measured the light reference spectrum for this assembly, $S_{t t}(\lambda)$ by inserting the light into the integrating sphere by the sample port entrance and collecting from the exit port of the sphere to the spectrometer. After that,

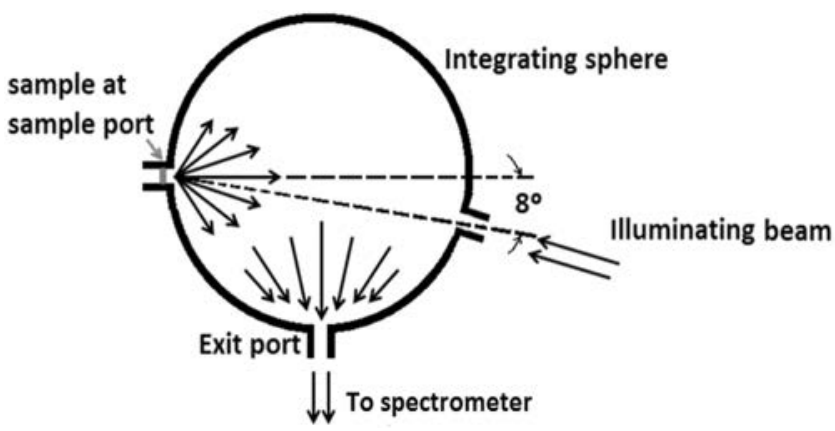

(b)

Fig. 1. Integrated measuring assemblies: (a) Total transmittance and (b) Total reflectance. 
the natural sample was placed at the entrance of the sample port of the integrating sphere and light was introduced into the sphere by crossing the sample. Total transmitted light is collected at the exit port of the sphere after being integrated inside the sphere and corresponding spectra were acquired using the scan mode of the spectrometer. After measuring the total transmitted spectrum of the natural sample, the optical clearing agent (EG or glucose $40 \%$ ) was added to the sample to start the treatment and measurements were made sequentially for a $30 \mathrm{~min}$ period. All the transmitted spectra measured from natural sample or under treatment, $T_{t t}(\lambda, t)$ were stored for later calculation of the total transmittance spectra using Eq. (1):

$$
T_{t}(\lambda, t)=100 \% \times \frac{T_{t t}(\lambda, t)}{S_{t t}(\lambda)} .
$$

The representation in Fig. 1(b) is the measuring assembly used to obtain total reflectance. Using this assembly, we started by measuring the corresponding reference spectrum, $S_{r t}(\lambda)$ in the same manner as in the total transmittance assembly. After that we placed the natural muscle sample at the entrance port of the sphere and illumination was then made through the illuminating port of the sphere at $8^{\circ}$ with the normal direction to the sample (right side of the sphere in Fig. 1(b)). With such configuration, the total reflected spectrum was measured from the natural sample, by collecting from the exit port of the sphere into the spectrometer. After measuring from the natural sample, the agent (EG or glucose 40\%) was added to the natural tissue sample to begin treatment and measurements were made sequentially for a $30 \mathrm{~min}$ period. All the reflected spectra measured from natural sample or sample under treatment, $R_{r t}(\lambda, t)$ were stored for later calculation of the total transmittance spectra using Eq. (2):

$$
R_{t}(\lambda, t)=100 \% \times \frac{R_{r t}(\lambda, t)}{S_{r t}(\lambda)} .
$$

\subsubsection{Nonintegrated measurements - collimated transmittance}

The nonintegrated measurements were made without the use of an integrating sphere. Figure 2 shows the simple assembly used to measure collimated transmittance: a collimated beam $(\varnothing=6 \mathrm{~mm})$ was

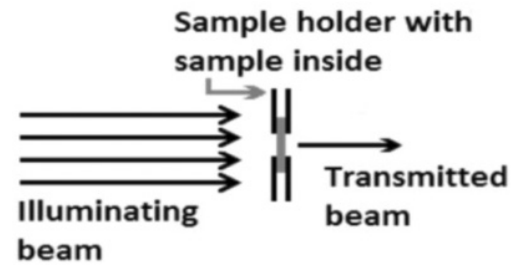

Fig. 2. Collimated transmittance measuring assembly.

directed normally to the sample. Immediately before and after the sample two pinholes $(\varnothing=1 \mathrm{~mm})$ were placed to reduce the beam diameter and to hold the sample inside the sample holder. The transmitted beam was measured on the opposite side of the sample.

Similarly to the previous measuring cases, we consider $S_{t c}(\lambda)$ as the spectrum of the light reference and $T_{t c}(\lambda, t)$ as the collimated transmitted spectrum measured from the sample at natural state or at a time $t$ of the treatment. Considering these spectra, we can calculate the collimated transmittance spectra for natural sample and for sample under treatment using Eq. (3):

$$
T_{c}(\lambda, t)=100 \% \times \frac{T_{t c}(\lambda, t)}{S_{t c}(\lambda)} .
$$

\subsubsection{Nonintegrated measurements - specular reflectance}

The measurement assembly for specular reflectance represented in Fig. 3 is accordingly with the total reflectance measurement assembly regarding angles and dimensions (Fig. 1(b)). It uses an incident beam at $8^{\circ}$ with the normal direction to the sample surface and the reflected beam is also measured at the same angular magnitude on the opposite side. A cross-section detail of the sample holder is represented at the left in Fig. 3. This sample holder is used in all the measuring assemblies described to ensure the same geometry in all measurements. As we can see from that detail, the sample is placed at the center of the sample holder and fixed laterally. When the treatments are applied, the agent (EG or glucose $40 \%$ ) is distributed above and below the sample creating sample immersion. The sample holder is completely sealed laterally by black walls to prevent the introduction of ambient light 


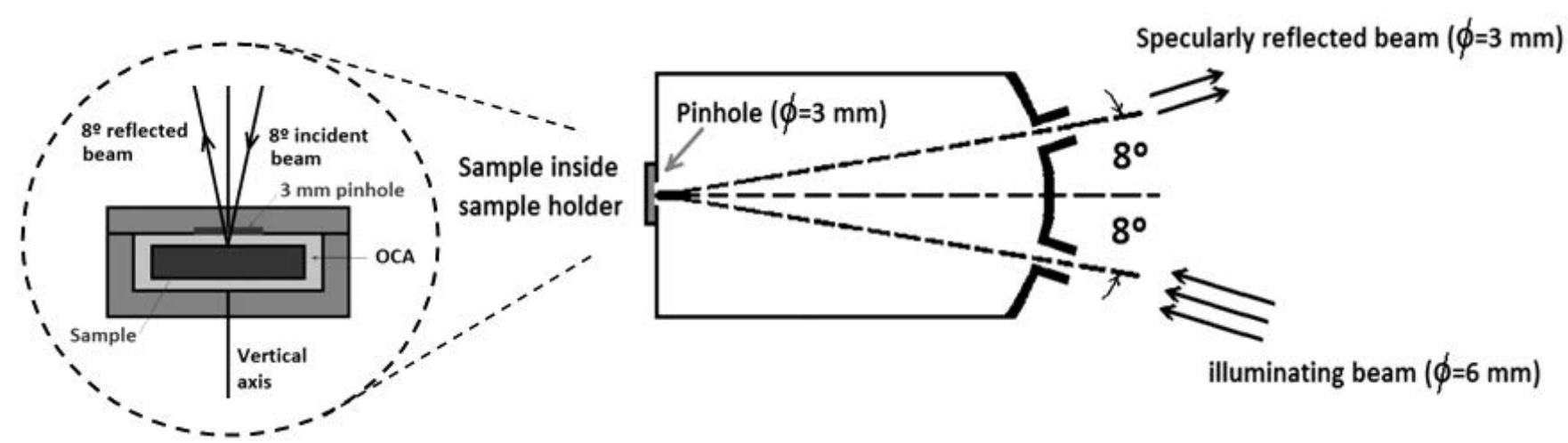

Fig. 3. Specular reflectance measuring assembly.

and below by a glass that allows for the entering of light from the source used in transmittance measurements.

The reference spectrum, $S_{s r}(\lambda)$, for the measuring assembly represented in Fig. 3 was acquired by placing a known reflecting reference material at the position of the sample holder and measuring the reflected spectrum with the spectrometer using the scan mode. Illumination was made at $8^{\circ}$ with central axis and the specularly reflected spectrum was acquired also at $8^{\circ}$ on the other side of the axis. After acquiring the reference spectrum, the reference reflecting material was replaced by the sample holder. The natural sample was placed inside the sample holder with its surface perpendicular to the axis of the assembly. Using the same measuring configuration as the one used when acquiring the reference spectrum the specularly reflected spectrum was measured from the natural sample. After that the agent (EG or glucose $40 \%$ ) was added to the sample and the immersion treatment began. Measurements of the specularly reflected spectra were made sequentially for a treatment period of $30 \mathrm{~min}$. The specularly reflected spectra, $R_{s r}(\lambda, t)$, measured from the natural sample and from the sample under treatment are used in Eq. (4) to calculate the specular reflectance spectra from the sample in natural state and at any time of the treatment:

$$
R_{s}(\lambda, t)=100 \% \times \frac{R_{s r}(\lambda, t)}{S_{s r}(\lambda)} .
$$

\subsubsection{Absorbance}

Considering the integrated measurements of total transmittance and total reflectance presented in Eqs. (1) and (2) and assuming that the light spectrum that is used in the integrated measurements is $100 \%$, the absorbance of the sample can be determined as the difference between $100 \%$ of light used and the integrated measurements. ${ }^{20}$

Equation (5) shows the calculation of sample's absorbance $(A)$ spectrum at a time $t$ during the optical clearing treatment:

$$
A(\lambda, t)=100 \%-\left[T_{t}(\lambda, t)+R_{t}(\lambda, t)\right] .
$$

\subsubsection{Diffuse reflectance}

Now considering total reflectance, we know that it contains both specular and diffuse terms. This way, using the measurements of total and specular reflectance (performed in the same conditions), we can use Eq. (6) to calculate diffuse reflection $\left(R_{d}\right)$ of the sample at time $t$ :

$$
R_{d}(\lambda, t)=R_{t}(\lambda, t)-R_{s}(\lambda, t) .
$$

The results obtained with these equations are represented in percentage of the light reference used in each case. Similarly to the absorbance calculations described above, the calculations made with Eq. (6) will also be necessary for latter estimation of the optical properties of the tissue.

We will now present the results obtained from the experimental measurements.

\section{Experimental Results}

In this section, we present the results obtained from the measurements, regarding the optical clearing treatments of muscle with the solutions of EG $99 \%$ and glucose $40 \%$. The results from the two studies will be presented side-by-side in the same figures for comparison in the following order: $T_{t}, T_{c}, R_{t}$ and $R_{s}$. In each particular case of measurement 


\section{Oliveira et al.}

and considering each of the two treatments we will present a figure containing parts (a) and (b):

- Part (a) of the figure corresponds to the treatment with EG. On the upper graph of part (a) we present the time dependence for the first minute with one second resolution for some particular wavelengths. The selected wavelengths are $400 \mathrm{~nm}, 500 \mathrm{~nm}, 600 \mathrm{~nm}, 700 \mathrm{~nm}, 800 \mathrm{~nm}, 900 \mathrm{~nm}$ and $1000 \mathrm{~nm}$. At the lower graph we present the time dependence for a treatment period of $30 \mathrm{~min}$ with one minute resolution after the third minute and for the same wavelengths. For better representation at the beginning of the treatment we have used $1 \mathrm{~s}$ resolution during the first minute, $10 \mathrm{~s}$ resolution during the second minute and $30 \mathrm{~s}$ resolution during the third minute.

- Part (b) of the figure contains similar representations to part (a), but now for the treatment with glucose $40 \%$.

Such representation in figures allows for immediate identification of the time dependence of the sample spectrum with the optical clearing treatment applied. The analysis of these figures and comparison between the two studies allows identifying differences between the two treatments. Also by recognizing some particularities for each of the two treatments we can identify and characterize the variation of the optical responses of the muscle sample with that treatment. Additionally, by analyzing the various figures we can take some conclusions regarding the variation of the optical

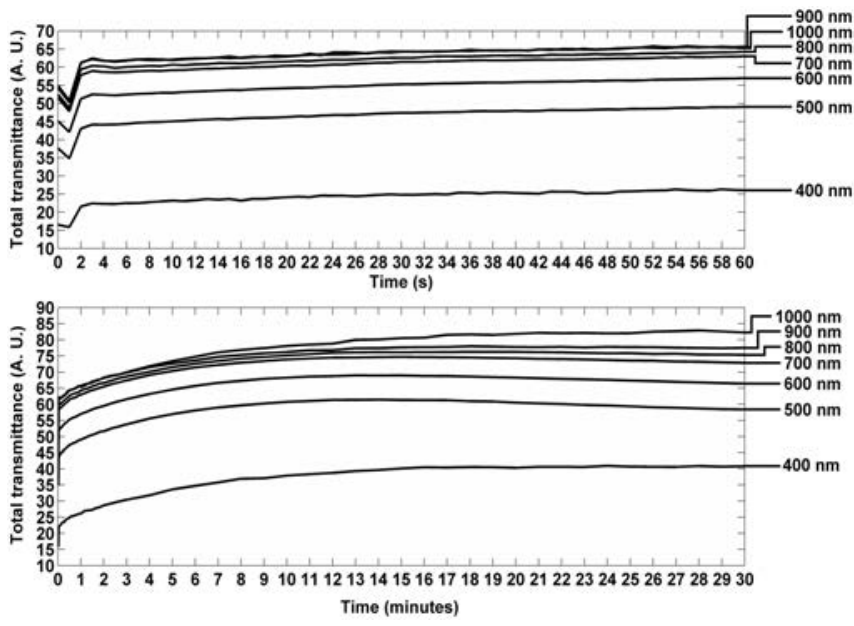

(a) properties of the tissue, even without making their estimation.

\subsection{Total transmittance}

From the total transmittance spectra calculated from the measurements made with the assembly represented in Fig. 1(a), we have retrieved the total transmittance values for each of the selected wavelengths and made the representations in Fig. 4.

As we can verify by comparing between both graphs related to the first minute of treatment (upper graphs in Fig. 4), the major changes in total transmittance are seen in the case of the treatment with EG. For this treatment (see upper graph in Fig. 4(a)) we see that total transmittance lowers a little in the first second of treatment. Such fact is probably caused by transit effects such as EG interaction with superficial layers of tissue in the course of filling up of the sample holder with the optical clearing agent (see detail on left of Fig. 3). After that, total transmittance rises significantly until $5 \mathrm{~s}$ of treatment, showing that the agent is already interacting with the superficial layers of the muscle sample and tissue water is going out. After $5 \mathrm{~s}$, total transmittance rises slower but close to linear on this short time interval, indicating that two oppositely directed fluxes-water out and agent into tissue-are formed. In the case of the treatment with glucose $40 \%$, we can see from the upper graph in Fig. 4(b) that the changes are much less significant than the ones observed in the treatment
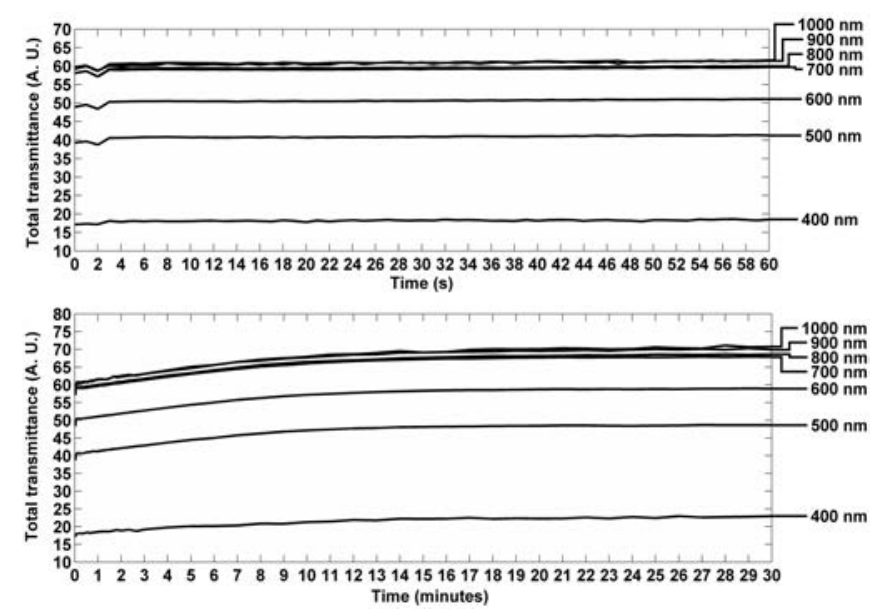

(b)

Fig. 4. Total transmittance evolution: (a) Treatment with EG for the first minute (upper graph) and for 30 min (lower graph), (b) Treatment with glucose $40 \%$ for the first minute (upper graph) and for $30 \mathrm{~min}$ (lower graph). 
with EG. This fact translates that glucose has a smaller osmotic pressure than the EG, since the aqueous solution used only contains $40 \%$ of glucose. Nevertheless the rise of the total transmittance can be observed, since the curves presented slowly rising behavior during the first minute of treatment.

Now considering the long-time variation (lower graphs in Fig. 4), we can see that the treatment with EG produces a higher rise in total transmittance than the treatment with glucose $40 \%$. This is verified for all the wavelengths selected, but the major difference is verified for the lower wavelengths, which indicates that EG is more powerful in improving total transmittance closer to ultraviolet. From the long-time evolution graphs in Fig. 4 we can identify the beginning of the saturation regime at approximately $10 \mathrm{~min}$ of treatment in both cases. In this regime the agent diffusion into the tissue is less intense than in the first minutes, since the tissue contains already a great amount of agent inside. For continuous agent diffusion into the tissue, the osmotic pressure that is maintained by the outside solution forces the tissue to maintain continuous dehydration so that by the leaving of water some space is available for the entering of more agent.

\subsection{Collimated transmittance}

As in the case of total transmittance, we have used the collimated transmittance spectra, calculated from the measurements made with the assembly

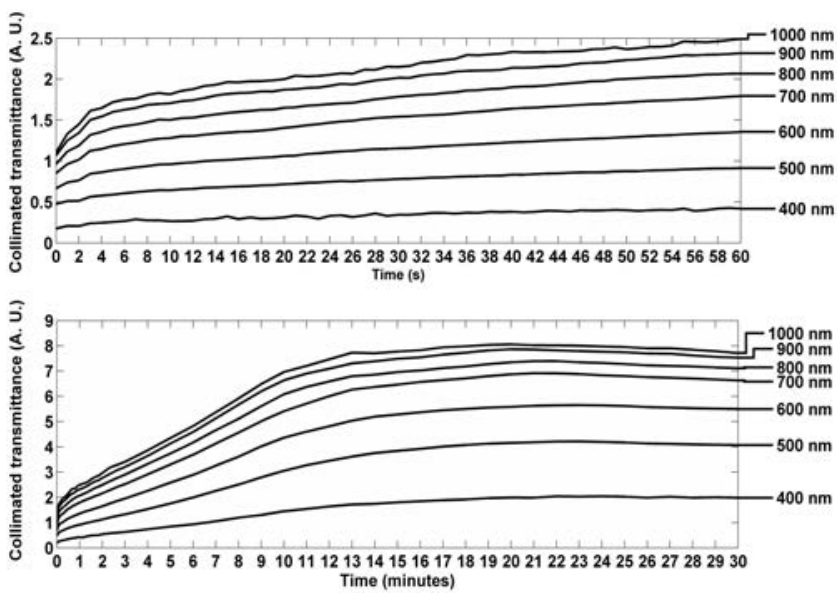

(a) represented in Fig. 2 to retrieve the $T_{c}$ values for the usual wavelengths. With these values we have made the representations in Fig. 5 to show the time evolutions in both treatments.

From the representations made in Fig. 5, we see higher magnitude variations than in the case of total transmittance (see Fig. 4). For the case of the treatment with EG, we observe a strong rise of collimated transmittance in the first $3 \mathrm{~s}$ (upper graph in Fig. 5(a)), while in the case of the treatment with glucose $40 \%$ that strong rise is observed in the first second of treatment (upper graph in Fig. 5(b)). This means that these two agents interact differently with the superficial layers of tissue. Additionally, we see that even after this initial strong increase in collimated transmittance, the diffusion of agents causes a gradually increasing behavior in collimated transmittance (EG - the rest of the first minute, and glucose after $18 \mathrm{~s}$ ). Such increasing behavior is stronger for the treatment with EG. The oscillation seen in the treatment with glucose for the first seconds probably indicates that tissue enters an initial stage of saturation and is forced to hydrate the superficial layers of the sample because of high concentration of water $(60 \%)$ in the solution, before allowing the diffusion of more agent into the tissue.

After analyzing the short-time evolution of collimated transmittance, we will now study the longtime variation for the treatments with EG and glucose $40 \%$. The lower representations in Fig. 5 show the long-time variations for both treatments.

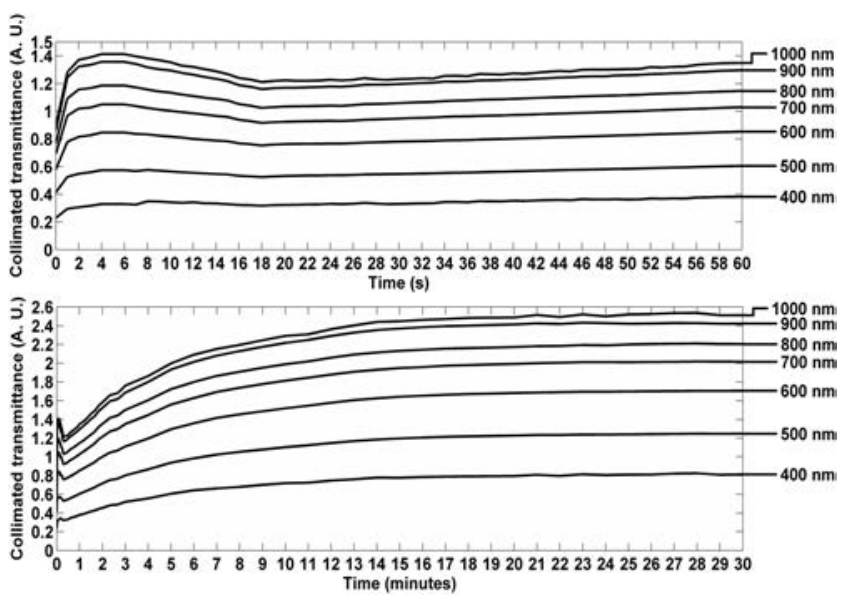

(b)

Fig. 5. Collimated transmittance evolution: (a) Treatment with EG for the first minute (upper graph) and for 30 min (lower graph), (b) Treatment with glucose $40 \%$ for the first minute (upper graph) and for 30 min (lower graph). 


\section{Oliveira et al.}

Analyzing these graphs, we can see that collimated transmittance rises in both cases of treatment, turning the tissue more transparent to light. For the case of EG (lower graph in Fig. 5(a)), we see that due to the strong diffusion power of this agent, collimated transmittance presents two stages of strong rising behavior: (i) strong initial rise in the first minute and (ii) less intense strong rising behavior between $1 \mathrm{~min}$ and $10 \mathrm{~min}$. After this initial period of strong rising behavior, collimated transmittance tends to slow its rising behavior between 10 and $20 \mathrm{~min}$ due to saturation of oppositely directed water and agent fluxes. A slight tissue "darkening" with time above $20 \mathrm{~min}$ could be caused by some impact of the agent on tissue. For instance, some tissue swelling may occur. ${ }^{16}$

For the case of the muscle sample treated with glucose $40 \%$ (lower graph in Fig. 5(b)), we observe that the major increase in collimated transmittance is confined to the first few minutes of treatment. This slowing in the rising behavior of $T_{c}$ precedes the saturation regime that starts at approximately 7 min.

Additionally, by comparing between lower graphs of Fig. 5 we can see that EG originates a greater increase in $T_{c}$ than glucose, around 8-fold for EG and only 3 -fold for glucose.

\subsection{Total reflectance}

The time dependencies represented in Fig. 6 correspond to the total reflectance variations for the usual

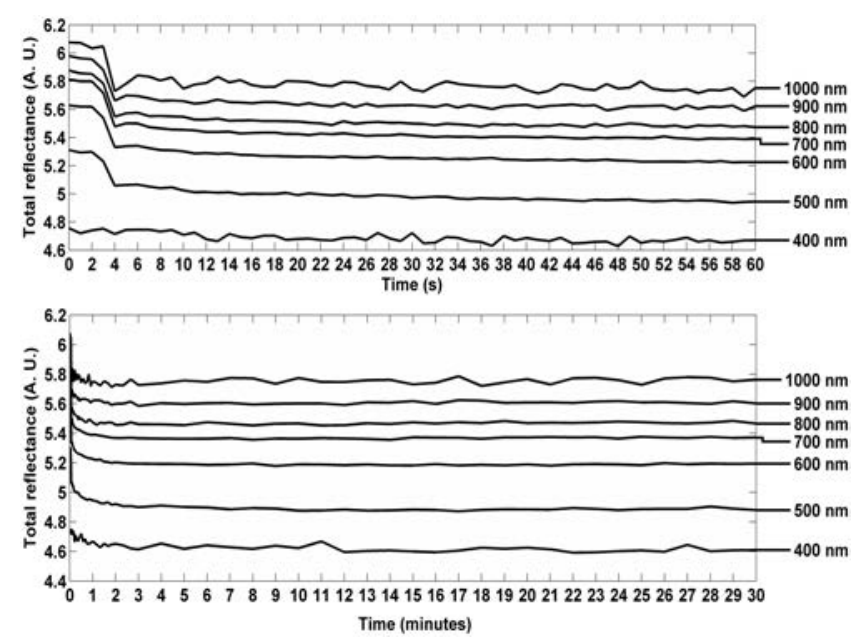

(a) wavelengths. Such values were retrieved from the $R_{t}$ spectra calculated from the original measurements made with the assembly represented in Fig. 1(b).

Analyzing and comparing between both upper graphs in Fig. 6, we see similar time dependence of $R_{t}$ in the first minute of treatment. For the treatment with EG (upper graph in Fig. 6(a)) there is a considerable decrease of $R_{t}$ in the first four seconds and after that a slow decreasing behavior. This behavior is also observed in the case of the sample treated with glucose $40 \%$ (upper graph in Fig. 6(b)). Since the resolution in the upper graphs presented in Fig. 6 is $1 \mathrm{~s}$, we can see various oscillations, especially for the extreme wavelengths $(400 \mathrm{~nm}$ and $1000 \mathrm{~nm})$.

Considering the long-time variation of $R_{t}$ (presented in both lower graphs of Fig. 6), we can see similarity of behavior between treatments. We can observe for both cases a major decrease in the first minute of treatment and after that $R_{t}$ tends to remain constant for the remaining of the treatment time. Although this similarity in behavior is observed in both treatments, different levels of $R_{t}$ are observed between cases.

\subsection{Specular reflectance}

As in the previous cases, we have retrieved the specular reflectance values for the usual wavelengths from the $R_{s}$ spectra calculated from the measurements obtained with the assembly represented in Fig. 3. The time evolutions for both treatments are represented in Fig. 7.

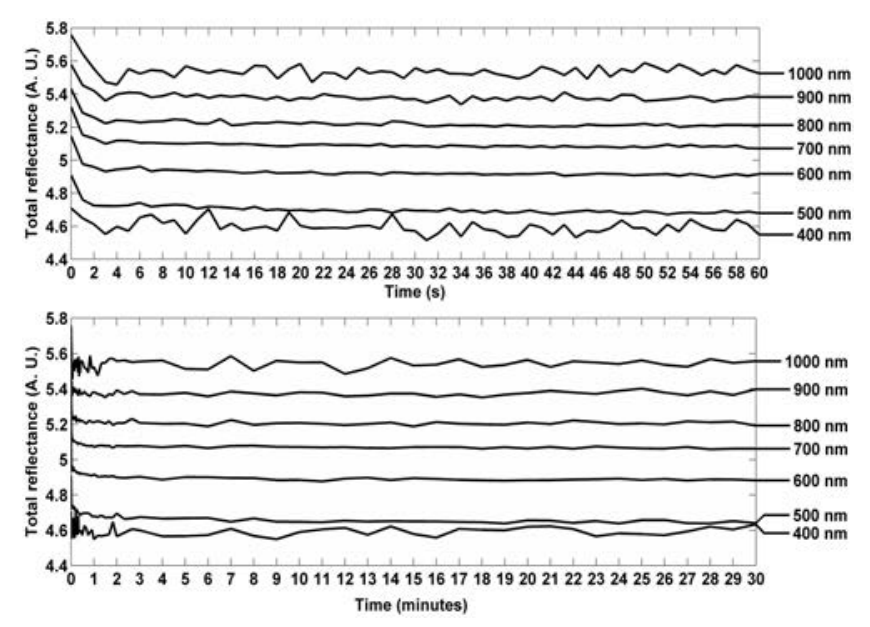

(b)

Fig. 6. Total reflectance evolution: (a) Treatment with EG for the first minute (upper graph) and for $30 \mathrm{~min}$ (lower graph), (b) Treatment with glucose $40 \%$ for the first minute (upper graph) and for $30 \mathrm{~min}$ (lower graph). 

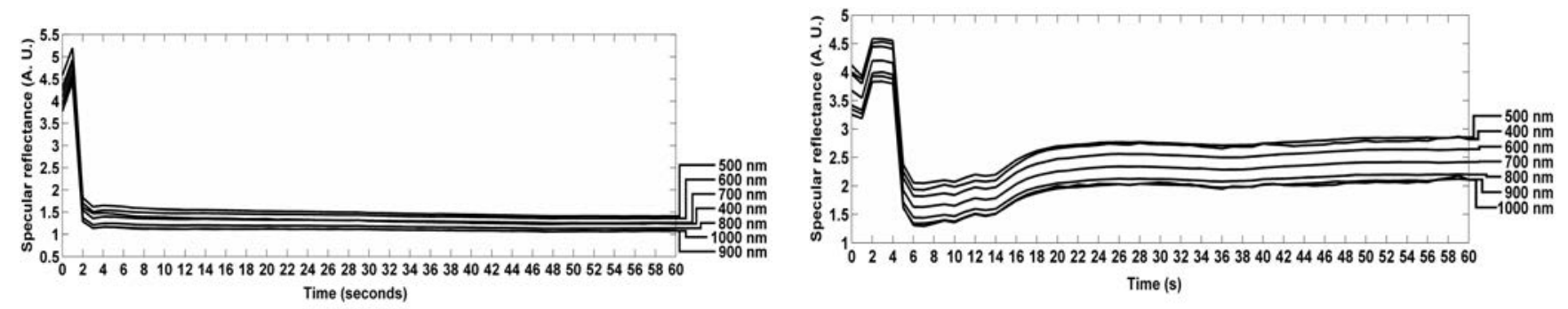

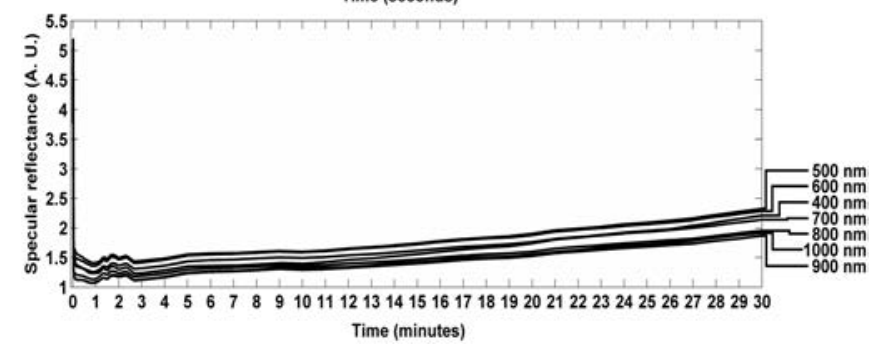

(a)

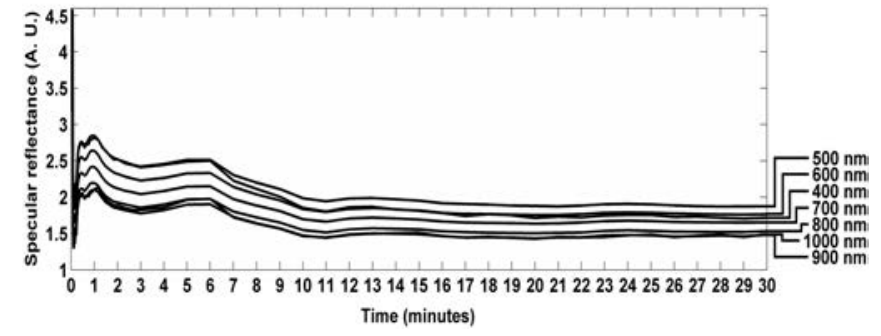

(b)

Fig. 7. Specular reflectance evolution: (a) Treatment with EG for the first minute (upper graph) and for 30 min (lower graph), (b) Treatment with glucose $40 \%$ for the first minute (upper graph) and for $30 \mathrm{~min}$ (lower graph).

In this case we observe different behavior between the treatments, both for short-time and for long-time representations. We will begin comparison between treatments considering the short-time evolutions of $R_{s}$. For the sample treated with EG (upper graph in Fig. 7(a)), we see an initial rise of $R_{s}$ in the first second, which is certainly related to the transit processes within the interfaces between tissue surface and OCA in the course of filling up the sample holder by the agent. At the beginning, when the solution is introduced in the sample holder an osmotic pressure is created over the tissue originating immediate water loss from the superficial layers. Additionally, when the agent is added to the tissue, it will fill up eventual tissue surface inhomogeneities providing the diminishing of tissue surface roughness. Both these effects might influence the initial increase of specular reflectance seen in upper graph of Fig. 7(a). After this initial rise, $R_{s}$ decreases strongly between 1 and $2 \mathrm{~s}$ of treatment. This means that during the short initial period of the treatment a large quantity of EG inserts into superficial layers of tissue sample. After that, a slow decreasing behavior can be observed after $6 \mathrm{~s}$, indicating that the lower tissue layers have a much lesser influence on the measured specular reflectance.

For the case of the treatment with glucose $40 \%$ (upper graph in Fig. 7(b)), we observe more or less similar behavior, besides a small decrease in $R_{s}$ in the first second of treatment, which can be related to above described transit processes at the initial interaction of OCA with tissue surface. Between 1 and $2 \mathrm{~s}, R_{s}$ rises, indicating less roughness of tissue and its superficial layers dehydration. Between 2 and $3 \mathrm{~s}, R_{s}$ remains almost unchanged. Between 3 and $4 \mathrm{~s}$ we see a major decrease in $R_{s}$, which indicates the entrance of considerable amount of glucose and possibly accompanied with tissue dehydration of upper layers. After this major decrease, we see a more stable behavior in $R_{s}$, between 4 and $14 \mathrm{~s}$, followed up by an increase observed between 14 and $20 \mathrm{~s}$. After that, the $R_{s}$ values tend to remain unchanged for the rest of the first minute.

Considering now the long time variation of $R_{s}$ represented in the lower graphs of Fig. 7, we can see some different behaviors between the two treatments: for the treatment with EG (lower graph in Fig. 7(a)), we see a major decrease in $R_{s}$ within the first minute. After the first minute, the saturation regime is observed with an increasing behavior, which indicates that the upper layers of the sample are losing some of the agent. However for that treatment, the final values of $R_{s}$ are still significantly lower than in natural state of the muscle sample. In the treatment with glucose $40 \%$ (lower graph in Fig. 7(b)), we see a decreasing behavior for $R_{s}$ that occurs in stages. First, we observe a major decrease within the first minute of treatment. Then, after the first minute and until the sixth 


\section{Oliveira et al.}

minute, $R_{s}$ slows its decreasing behavior and tends to stabilize, indicating that the upper layers of tissue are almost saturated with agent. We see another decreasing behavior between the sixth and the tenth minutes of treatment. This indicates that the osmotic pressure of glucose has risen to diffuse some more agent into the superficial tissue layers. After the tenth minute of treatment, we observe the final saturation regime by the stability of the $R_{s}$ levels in all curves.

The decreasing behavior seen from the lower graphs in Fig. 7 can be justified if we consider the various interfaces crossed by light in measuring $R_{s}$ and the refractive index mismatch at the interfaces. From the detail presented at the left side of Fig. 3, we see that when performing these measurements, light crosses several interfaces between media (air-OCA-tissue-OCA-air). During optical clearing, agent enters the tissue, causing an increase in the refractive index of the tissue and also a decrease in tissue surface roughness. On the other hand tissue dehydrates, causing a decrease in the refractive index of the surrounding media: outside OCA dilutes in water that leaves the tissue. Both these mechanisms should give a tendency of specular reflectance increase. Due to sample surface roughness and light beam penetration into scattering tissue, scattering plays a significant role when measuring specular reflectance. At first minutes of agent diffusion into top layers of tissue and its dehydration, partial refractive index matching is obtained near the interface between tissue and OCA and tissue surface roughness is reduced. As a consequence, scattering at the interface and top layers of tissue reduces leading to a significant decrease in $R_{s}$ measurements during the first minutes of optical clearing as we can see from lower graphs in Fig. 7. However, refractive index mismatch still exists at the interface between tissue and OCA and it will increase at optical clearing. This fact can be verified by the slight increase in $R_{s}$ graph for EG treatment (lower graph in Fig. 7 (a)) after the first minute. ${ }^{17,21}$ For the case of glucose, due to the presence of $60 \%$ of water in the solution, which is approximated to tissue water content $(75.6 \%)$, the above described effects cannot be very significant, as we observe by lower graph in Fig. 7(b).

It should be noted that in spite of some differences in EG and glucose interaction with the muscle tissue, the general behavior of the specular reflectance is similar, because it depends mostly on Fresnel reflection from OCA/tissue interface and scattering by tissue rough surface and by superficial and upper layers of the tissue. Thus we have found a very fast reflectance decay (2-fold) for EG during $\sim 1.5 \mathrm{~s}$ and for glucose during $\sim 5 \mathrm{~s}$, caused by a fast permeability of tissue for a smaller depth, ${ }^{16}$

$$
\tau=\frac{d^{2}}{D_{a}}
$$

where $\tau$ is the characteristic diffusion time through a tissue layer of thickness $d$ for an OCA diffusing through one surface of the sample with the diffusion coefficient $D_{a}$ in the particular kind of tissue and

$$
D_{a}=\text { const. } \times M^{-S},
$$

where $M$ is the molecular mass of OCA, and parameter $S$ depends on molecular form and size. For diffusion in water, $S$ is approximately 0.5 for spherical small molecules and approximately 0.3 for bigger protein molecules.

If we suppose that glucose diffusion coefficient in dermis is close to its value in muscle tissue, we can take experimental data for $D_{G}=2.5 \times 10^{-6} \mathrm{~cm}^{2} / \mathrm{s}$, thus for experimental value of $\tau_{G} \cong 5 \mathrm{~s}$ (upper graph of Fig. 7(b)), we can use Eq. (7) to find tissue thickness of superficial tissue layer, which has a strong impact on initial specular reflectance as $d \cong 35.4 \mu \mathrm{m}$.

In the case of EG interaction with muscle tissue, we can suppose that the water flux directed at tissue dominates in the initial stage due to the osmotic stress caused by highly concentrated EG and its high viscosity. This way in accordance with Eq. (8), $D_{\mathrm{H}_{2} \mathrm{O}}$ is expected as

$$
D_{\mathrm{H}_{2} \mathrm{O}} \cong D_{G}\left(M_{G} / M_{\mathrm{H}_{2} \mathrm{O}}\right)^{1 / 2} .
$$

Thus, because of molecular mass for glucose is 180 and for $\mathrm{H}_{2} \mathrm{O}$ is only 18 , we will have 3.2fold bigger diffusivity of water molecules then glucose molecules. For experimental value of $\tau_{E G} \cong$ $1.5 \mathrm{~s}$ (upper graph in Fig. $7(\mathrm{a})), d \cong 34.6 \mu \mathrm{m}$, i.e. excellent fit to above estimations for glucose diffusion.

From the experimental data obtained from $T_{c}$ measurements for each of the wavelengths represented in the lower graphs of Fig. 5, we can perform a similar analysis to obtain the diffusion time $(\tau)$ and diffusion coefficients of both agents $\left(D_{a}\right)$, EG and glucose. ${ }^{16}$ Considering the $30 \mathrm{~min}$ of 


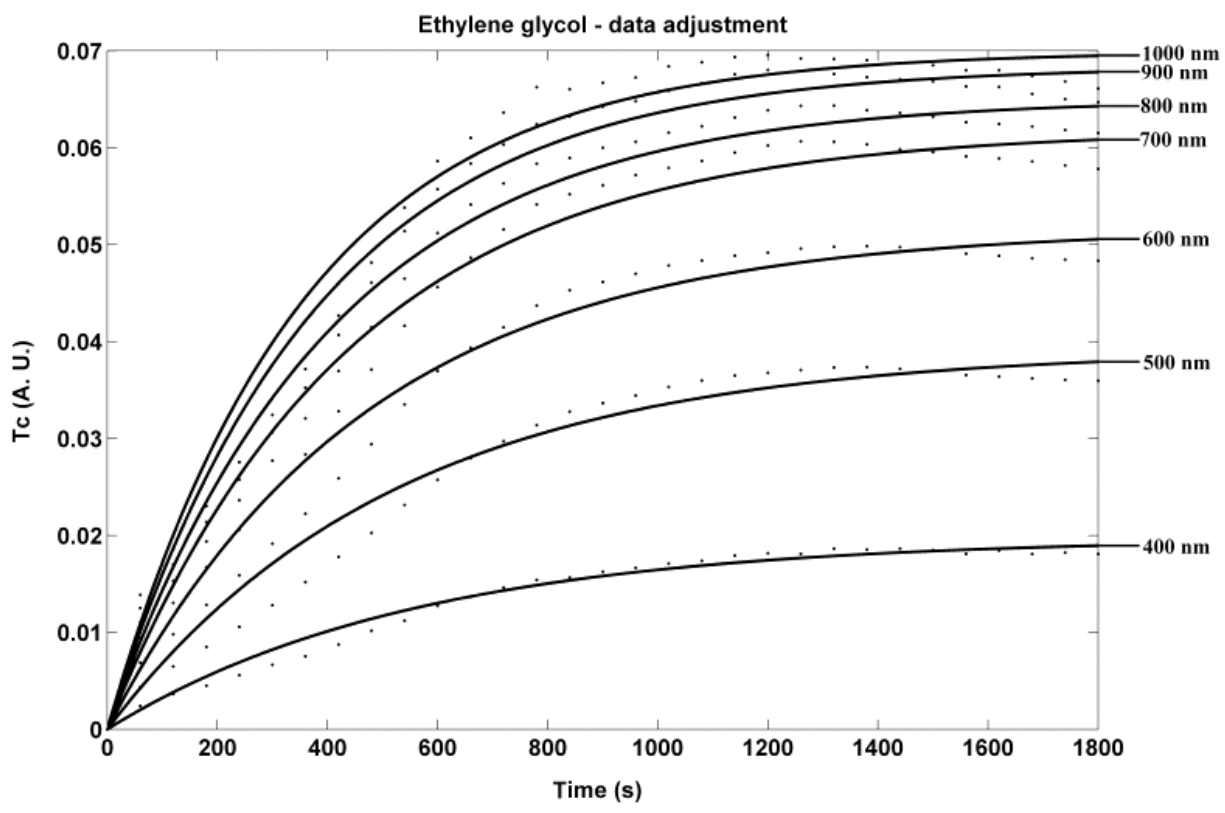

Fig. 8. EG $T_{c}$ experimental data fitting using Eq. (10).

treatment and the values of $T_{c}$ measurements made in the treatment with EG (lower graph in Fig. 5(a)), we have fitted the experimental data to curves with equations like the one in Eq. (10), which is valid for agent diffusion from the both surfaces of the sample ${ }^{16}$ :

$$
\begin{gathered}
T_{c}(\lambda, t)=a(\lambda) \times\left[1-\exp \left(-\frac{t}{\tau(\lambda)}\right)\right], \\
\tau=\frac{d^{2}}{\pi^{2} \times D_{a}} .
\end{gathered}
$$

For the case of EG, we have obtained the following fitting curves (Fig. 8) for each of the usual wavelengths.

Considering the adjustments made for each wavelength represented in Fig. 8, we have obtained the average $\tau=445.0 \mathrm{~s}$ and corresponding standard deviation (74.9) for the treatment with EG. With this value for the diffusion time and with the thickness value that we have measured for this time of treatment from a muscle sample treated with EG, we have used Eq. (11) to determine the diffusion coefficient of EG into muscle. This calculation is presented in Eq. (12):

$$
\begin{aligned}
D_{\mathrm{EG}} & =\frac{d_{\mathrm{EG}}^{2}}{\pi^{2} \times \tau_{\mathrm{EG}}}=\frac{0.05^{2}}{\pi^{2} \times 445.0} \\
& =5.6 \times 10^{-7} \mathrm{~cm}^{2} / \mathrm{s} .
\end{aligned}
$$

Using a similar procedure, we have made fittings to the $T_{c}$ measurements for the treatment with glucose
$40 \%$ (lower graph in Fig. 5(b)) to calculate the mean $\tau$ value for that treatment. ${ }^{16}$ The fitting curves for glucose treatment are presented in Fig. 9 for each of the usual wavelengths according to Eq. (10).

Considering the adjustments represented in Fig. 9 for the various wavelengths, we have calculated the mean $\tau=303.0 \mathrm{~s}$ and corresponding standard deviation (17.4). For this value of the diffusion time of glucose and considering the sample thickness measured at this time of treatment from a muscle sample under treatment with glucose $40 \%$, we have used Eq. (11) to determine the diffusion coefficient of glucose into muscle. This calculation is presented in Eq. (13):

$$
\begin{aligned}
D_{G} & =\frac{d_{G}^{2}}{\pi^{2} \times \tau_{G}}=\frac{0.05^{2}}{\pi^{2} \times 303.0} \\
& =8.3 \times 10^{-7} \mathrm{~cm}^{2} / \mathrm{s} .
\end{aligned}
$$

Considering the diffusion coefficient in skeletal muscle obtained for the case of glucose $40 \%$, we must say that it is in good agreement to the one obtained for glucose in skin dermis $\left(D_{G}=\right.$ $\left.2.5 \times 10^{-6} \mathrm{~cm}^{2} / \mathrm{s}\right) \cdot{ }^{16}$ On the other hand, we can see that EG presents a lower diffusion coefficient than glucose $40 \%$ in skeletal muscle. Considering that the EG solution is almost pure (99\% of EG and $1 \%$ of water), we can understand and justify this fact. First, it has been verified by other 


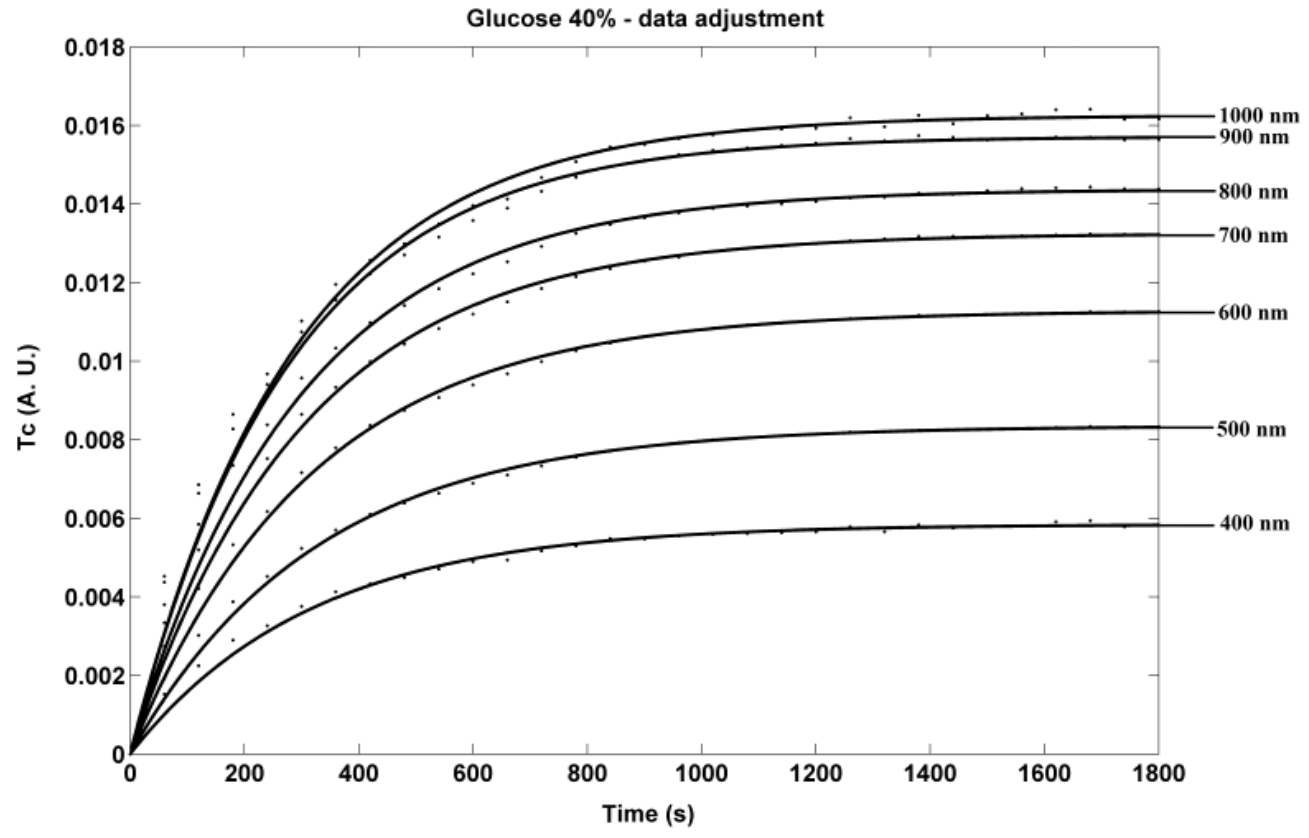

Fig. 9. Glucose $T_{c}$ experimental data fitting using Eq. (10).

authors $^{19}$ that glucose diffuses faster into biological tissues than for instance glycerol, even its molecular mass is a few times lower than for glucose. In this study, Cicchi et al. have used glycerol in anhydrous form and glucose in aqueous solution $5 \mathrm{M}$. The molecular mass of glycerol is $92 \mathrm{~g} / \mathrm{mol}$. These authors also have observed that in spite of diffusing faster, it creates a less efficient optical clearing effect than glycerol, which is also our case when comparing the magnitude of the optical clearing effects of EG and glucose (compare between both lower graphs of Fig. 5 - higher increase in $T_{c}$ for EG than for glucose 40\%). Such high diffusion speed and less efficient optical clearing effect created by glucose than glycerol is certainly imposed by the difference in viscosity of the two agents. In our case and for $25^{\circ} \mathrm{C}$, we have for the glucose solution a viscosity coefficient of $1 \times 10^{-3}$ N.s.m ${ }^{-2}$ (Ref. 22), and for EG the viscosity coefficient is $1.61 \times 10^{-2}$ N.s.m ${ }^{-2}$ (Ref. 23) (16.1-fold of glucose). Due to these values of viscosity coefficients, we can understand why EG shows a smaller value for the diffusion coefficient and a higher diffusion time than glucose - EG has more difficulty in entering the muscle and so takes more time to do so.

In the case of EG treatment, since the solution has a high concentration of EG, we verify an initial and strong osmotic pressure caused by EG onto the upper layers of skeletal muscle (see lower graph of
Fig. (5(a)) and tissue upper layers are forced to dehydrate fast. We can see such fast dehydration by the fast decrease seen in $R_{s}$ measurements (top graph in Fig. 7(a)). After dehydration of the tissue upper layers, and due to its considerable high viscosity, EG has more difficulty in diffusing into tissue and so it diffuses slower. This indicates that EG diffusion is done in two stages with different velocities for EG in skeletal muscle.

On the other hand for the treatment with glucose $40 \%$, the effect of viscosity is not so perceptible, since this solution has a considerable smaller viscosity coefficient. Also, the solution contains $40 \%$ of glucose and $60 \%$ of water, which is similar to the content of water in the tissue $(75.6 \%) .{ }^{12}$ Such similar values of water content in tissue and solution are impeditive to observe a fast dehydration of the skeletal muscle, which is verified by the slow decrease in $R_{s}$ measurements for this treatment (see upper graph of Fig. 7(b)). The initial diffusion of glucose into the top layers of the muscle is done in a smaller time period than the diffusion of EG, and for glucose no two-stage diffusion is seen (lower graph on Fig. 5(b)).

\section{Discussion of Results}

As we could observe from the various representations in Sec. 3, the non-integrated measurements 
are preferable to detect the variations caused by the optical clearing treatments applied than the integrated measurements - greater magnitude variations are observed. Considering all the results presented and the wavelengths selected for the representations, we could see bigger clearing efficiency for shorter wavelengths, where scattering dominates in the natural tissue.

From the collimated transmittance variations, we could see that EG creates a higher magnitude optical clearing effect than glucose, but it takes more time to achieve the saturation regime. It was also possible to verify that the strong initial rising behavior of collimated transmittance is organized in two stages for EG, while in the case of the treatment with glucose $40 \%$ it has only one initial stage of strong rising. These strong rising behaviors seen in collimated transmittance translate the two mechanisms of optical clearing: tissue dehydration and agent inclusion inside the tissue. For the case of glucose we do not see a decreasing behavior during the saturation regime, but in the case of EG such decreasing is seen, which translates impact of EG on tissue and the occurrence of some tissue swelling. Apart from these details in the behavior, the collimated transmittance values seen at the end of the treatments is significantly higher than the values observed for the natural tissues. Such fact indicates a great loss of light scattering during the treatments. A better organized structure inside the tissue must be achieved during the $30 \mathrm{~min}$ period of the treatments applied. The loss of scattering is due to the refractive index matching that occurred due to the loss of water and consecutive tissue impregnation by the agent (EG or glucose).

The variations observed for specular reflectance and diffuse reflectance complement these observations, although some transient effects were observed due to the deposition of agent at the superficial layers of the tissues and consequent elimination of superficial inhomogeneities.

An interesting case was observed in the measurements of specular reflectance for the treatment of glucose $40 \%$, where some initial oscillations were detected. Such oscillations indicate the immediate impact of glucose on the upper layers of the tissue. The osmotic pressure created by the outside solution induces the loss of water from the outermost layers of the tissue followed by some agent flux into these layers and consecutive hydration of the layers from the inside to compensate for the unbalanced water content between outside layers of tissue and surrounding solution. Such oscillations are only seen in the treatment with the solution of glucose. For the case of EG they are not seen because of the high concentration that EG presents in solution (99\%).

Due to the experimental assembly used to measure specular reflectance (Fig. 3), we see that in this measuring procedure light traverses the interface between OCA and tissue to interact with the top layer of tissue. Due to tissue surface roughness, refractive index mismatch at the interface and light beam penetration into tissue, there is a great contribution of light scattering in the specular reflected spectra. During tissue treatment, there are two leading mechanisms involved - agent diffusion into the tissue and tissue dehydration. The first mechanism causes refractive index matching at top layers of the tissue and reduction in tissue surface roughness. The second mechanism leads to refractive index matching of upper tissue layers and eliminate inclusion of tissue bulk scattering into specular reflection. It also can induce a slight decrease of the refractive index of the solution around the tissue. As a consequence the measured specular reflectance decreases greatly in the first minute of the treatment, as we can see in the lower graph of Fig. 7(a) for the case of EG and then slowly goes up. In the case of the treatment with glucose, the phenomenon is the same, but due to a lower concentration of glucose in solution and also to a similar content of water in outside solution and natural tissue, the initial decrease in specular reflectance (lower graph in Fig. $7(\mathrm{~b})$ ) is not so intense and so fast as in the case of the treatment with EG. Such scattering contamination in the specular reflectance measurements is important at the first minute of treatment with EG and at the first $12 \mathrm{~min}$ of the treatment with glucose, but later on, scattering can be neglected. This way, we are able to see the specular reflectance behavior after $1 \mathrm{~min}$ (EG) or $12 \mathrm{~min}$ (glucose). We observe that due to high concentration of EG in solution, specular reflectance increases linearly (slowly) after $1 \mathrm{~min}$ of treatment. For the case of glucose and since the concentration of glucose in solution is considerably lower than the one for EG, specular reflectance remains almost unchanged since $12 \mathrm{~min}$ of treatment elapsed. 


\section{Conclusion}

From the variations observed in the different measurements and calculations made for the treatments with EG and glucose, we were able to identify that the optical clearing effects reduce light scattering inside the muscle. From the analysis of the collimated transmittance variations, we could estimate the characteristic diffusion times and diffusion coefficients of EG and glucose in the muscle, which are necessary to calculate the variation of the refractive index profile of the tissue during treatment. Such variation of the refractive index profile of the muscle is necessary to the inverse Monte Carlo simulations to estimate the variation of the optical properties of the tissue during the treatments.

By comparing the several representations in Sec. 3, we can say that collimated transmittance is the optimal measurement procedure to demonstrate the optical clearing efficacy of the tissue. As a complement to the information retrieved from these measurements, some characteristics and information could be retrieved from the measurements of specular reflectance. In particular the identification of transient effects was retrieved from these representations. Additionally, we could identify some scattering contamination in the reflection measurements and calculations due to changing of agent concentration in outside solution as a consequence of tissue dehydration and Fresnel reflections at interfaces existent in the measurements of total reflectance and specular reflectance.

To proceed with our research and use the measurements presented above in the estimations of the variation of the optical properties of the muscle under treatment, we must first perform some corrections to eliminate this scattering contamination in the measurements of specular reflectance.

We will use the corrected results later to estimate the variations of the optical properties of muscle under treatment with these solutions. From the increase in collimated transmittance and decrease in specular reflectance that we have observed in both cases we can estimate that the scattering coefficient will decrease with the applied treatments. From the observations made with the measurements of collimated transmittance, we saw that the treatments induce an increase in transmittance. Such increase is caused by a reduction in the scattering coefficient and due to an increase in the $g$ factor.

\section{Acknowledgments}

The authors would like to thank the following institutions for all the help in preparation of the samples and resources made available to perform the measurements: CIETI - Centro de Inovação em Engenharia e Tecnologia Industrial, ISEP Instituto Superior de Engenharia do Porto, Portugal. LAIMM — Laboratório de Apoio à Investigação em Medicina Molecular, Departamento de Biologia Experimental, Faculdade de Medicina da Universidade do Porto, Portugal.

This work was also supported in part by grants: RF Governmental contracts 11.519.11.2035, 14 . B37.21.0728, and 14.B37.11.0563; FiDiPro, TEKES Program (40111/11), Finland; SCOPES EC, Uzb/ Switz/RF, Swiss NSF, IZ74ZO_137423/1; RF President's grant "Scientific Schools", 1177.2012.2.

\section{References}

1. V. V. Tuchin, M. Leahy, D. Zhu, "Introduction special issue: Optical clearing for biomedical imaging," J. Innov. Opt. Health Sci. 3(3), v-vi (2010).

2. C. Liu, Z. Zhi, V. V. Tuchin, Q. Luo, D. Zhu, "Enhancement of skin optical clearing efficacy using photo-irradiation," Lasers Surg. Med. 42, 132-140 (2010).

3. A. N. Bashkatov, E. A. Genina, V. V. Tuchin, "Optical properties of skin, subcutaneous, and muscle tissues: A review," J. Innov. Optic Health Sci. 4(1), 9-38 (2011).

4. S. Plotnikov, V. Juneja, A. B. Isaacson, W. Mohler, P. Campagnola, "Optical clearing for improved contrast in second harmonic generation imaging of skeletal muscle," Biophys. J. 90(1), 328-339 (2006).

5. R. laComb, O. Nadiarnykh, S. Carey, P. Campagnola, "Quantitative second harmonic generation imaging and modeling of the optical clearing mechanism in striated muscle and tendon," J. Biomed. Opt. 13(2), 21,109-21,111 (2008).

6. N. Sudheendran, M. Mohamed, M. G. Ghosn, V. V. Tuchin, K. V. Larin, "Assessment of tissue optical clearing as a function of glucose concentration using optical coherence tomography," J. Innov. Opt. Health Sci. 3(3), 169-176 (2010).

7. X. Wen, S. L. Jacques, V. V. Tuchin, D. Zhu, "Enhanced optical clearing of skin in vivo and OCT in depth imaging," J. Biomed. Opt. 17(6), 066022-1-066022-6 (2012).

8. C. G. Rylander, O. F. Stumpp, T. E. Milner, N. J. Kemp, J. M. Mendenhall, K. R. Diller, A. J. Welch, 
"Dehydration Mechanism of optical clearing in tissue," J. Biomed. Opt. 11(4), 041117-1-041117-7 (2006).

9. L. Oliveira, "Study of the spectral transmission response of biological tissues under the influence of different osmotic agents," M.Sc. thesis, FEUP Faculdade de Engenharia da Universidade do Porto, Porto, Portugal (2007).

10. X. Wen, Z. Mao, Z. Han, V. V. Tuchin, D. Zhu, "In vivo skin optical clearing by glycerol solutions: Mechanism," J. Biophotonics 3(1-2), 44-52 (2010).

11. G. Vargas, E. K. Chan, J. K. Barton, H. G. Rylander III, A. J. Welch, "Use of an agent to reduce scattering in skin," Lasers Surg. Med. 24, 133-141 (1999).

12. R. F. Reinoso, B. A. Telfer, M. Rowland, "Tissue water content in rats measured by desiccation," J. Pharmacol. Toxicol. Meth. 38(2), 87-92 (1997).

13. L. Oliveira, A. Lage, M. Pais Clemente, V. V. Tuchin, "Rat muscle opacity decrease due to the osmosis of a simple mixture," J. Biomed. Opt. 15(5), 055004-1-055004-9 (2010).

14. L. Oliveira, A. Lage, M. Pais Clemente, V. V. Tuchin, "Optical characterization and composition of abdominal wall muscle from rat," Opt. Lasers Engrg. 47(6), 667-672 (2009).

15. L. Oliveira, A. Lage, M. Pais Clemente, V. V. Tuchin, "Concentration dependence of the optical clearing effect created in muscle immersed in glycerol and ethylene glycol," SPIE Proceedings of Saratov Fall Meeting - 2006, Vol. 6535, V. V. Tuchin Ed. (2007).

16. V. V. Tuchin, Optical Clearing of Tissues and Blood, SPIE Press, Bellingham, WA (2006).

17. E. A. Genina, A. N. Bashkatov, V. V. Tuchin, "Tissue optical immersion clearing," Expert Rev. Med. Devices 7(6), 825-842 (2010).

18. J. Hirshburg, B. Choi, J. S. Nelson, A. T. Yeh, "Collagen solubility correlates with skin optical clearing," J. Biomedical Optics 11(4) (2006).

19. R. Cicchi, F. S. Pavone, D. Massi, D. D. Sampson, "Contrast and depth enhancement in two-photon microscopy of human skin ex vivo by use of optical clearing agents," Optics Express 13(7), (2005).

20. M. Bass, V. N. Mahajan, E. Van Stryland, Handbook of Optics - Vol. II, McGraw Hill (2010).

21. E. A. Genina, A. N. Bashkatov, A. A. Korobko, E. A. Zubkova, V. V. Tuchin, I. Yaroslavsky, G. B. Altshuler, "Optical clearing of human skin: Comparative study of permeability and dehydration of intact and photothermally perforated skin," J. Biomed. Opt. 13(2), 021102 (2008).

22. A. Converti, M. Zilli, S. Arni, R. Di Felice, M. Del Borghi, "Estimation of viscosity of highly viscous fermentation media containing one or more solutes," Biomed. Engrg. J. 4, 81-85 (1999).

23. G. Elert, The Physics Hypertexbook - Viscosity, http://hypertextbook.com/physics/matter/ viscosity/ (2007). 